\title{
Prioridade Dinâmica de Mensagens Aplicada a Redes de Sensores Corporais Sem-Fio
}

\author{
Felipe da Rocha Henriques \\ felipe.henriques@cefet-rj.br \\ CEFET/RJ-Campus Petrópolis \\ Raphael Guedes \\ raphael.guedes@estacio.br \\ Universidade Estácio de Sá
}

\author{
Creyton Ferreira \\ creyton.ferreira@aluno.cefet-rj.br \\ CEFET/RJ-Campus Petrópolis \\ Laura Silva de Assis \\ laura.assis@cefet-rj.br \\ CEFET/RJ-Campus Petrópolis
}

\begin{abstract}
The Wireless Body Area Networks (WBANs) are a special case of the Wireless Sensor Networks (WSNs) and, in general, are responsible for gathering and transmitting biometric data from a given patient. One of the features of the WSNs is the self-configuration; i.e., the capability that a given parameter can be adjusted regarding some occurrence in execution time. As stated, the objective of the WBANs is the gathering, through several sensors, of biometric data of a patient. In general, because of possible daily activities and/or pathologies, some sensors can be more demanded (or required) in certain periods of the day. In these situations, information of these mentioned sensors are more relevant and require larger data delivery priority. Thus, we propose QoSBody-AODV, a variation of the AODV routing protocol, which performs the dynamic adjustment of the message's priority, turning the network functioning to be sensitive to the physical activities performed by the patient, as well as pathological information.
\end{abstract}

\section{KEYWORDS}

Redes de Sensores Sem fio, Redes de Área Corporal Sem Fio, QoS, Energia, AODV.

\section{INTRODUÇÃO}

As Redes de Sensores Sem-Fio (RSSFs) são um tipo particular de redes ad hoc, formadas por dispositivos sensores que possuem capacidade de coletar dados em diferentes contextos, podendo, de maneira autônoma, transmiti-los através de um padrão de rede sem-fio apropriado [1] [2]. Os sensores são dispositivos com capacidades restritas de processamento, comunicação e energia. Além disso, uma das características importantes das RSSFs é a sua capacidade de auto-configuração; ou seja, a depender da aplicação (contexto onde a rede se insere), os seus nós (sensores) devem ser capazes de se configurarem de forma a prover um monitoramento adequado, com a maior autonomia possível.

Em geral, esses dispositivos são formados por quatro unidades básicas: sensoriamento, processamento, comunicação e uma unidade de energia. A unidade de sensoriamento é responsável por capturar os eventos do ambiente, e converter os sinais analógicos produzidos em sinais digitais. A unidade de processamento recebe os dados digitais e realiza as operações necessárias, de acordo com o objetivo da aplicação. Além disso, essa unidade gerencia as tarefas a serem realizadas e enviadas para outro sensor da rede. Os dados, quando prontos, são levados à camada de comunicação, responsável pela transmissão e recepção de mensagens na rede. Por fim, temos unidade de energia, responsável por manter o sensor ativo

As Redes de Sensores Corporais Sem-Fio (Wireless Body Area Network - WBANs) são um tipo de RSSF, cujos dispositivos coletam dados biométricos de um indivíduo, tais como eletrocardiograma (ECG) [3], eletroencefalograma (EEG), saturação de oxigênio no sangue ou até os movimentos de membros. A comunicação é tipicamente sem-fio, onde dois padrões comumente usados são: o IEEE 802.15.4 [4] e o IEEE 802.15.6 [5].

Nesse âmbito, este trabalho apresenta o algortimo QoSBodyAODV, uma variação do protocolo de roteamento AODV (Ad-hoc On Demand Distance Vector) [6] que busca uma maior confiabilidade da rede, que traduziremos aqui como qualidade de serviço (QoS), tornando o seu funcionamento sensível ao contexto clínico no qual o paciente encontra-se submetido a suas atividades cotidianas. Desse modo, propondo uma análise mais realista, este trabalho estende os conceitos propostos inicialmente em [7] e [8], de modo a avaliar a rede através de situações clínicas e cotidianas específicas, como o caso de um paciente com problemas cardiovasculares, por exemplo.

A título de exemplo, pode-se imaginar um paciente que encontrase sob supervisão clínica. O médico, no que lhe concerne, solicita medições por todo o período de um dia típico do paciente; ou seja, isto engloba os períodos de estados como dormindo, praticando atividades físicas e/ou atividades do dia-a-dia. Nesse contexto, de acordo com o período do dia e/ou atividade do paciente, alguns sensores serão mais exigidos que outros. Por exemplo, quando um paciente inicia uma atividade física, os dados transmitidos pelos sensores (ou sensor) relacionados à circulação cardiovascular, precisam ter uma prioridade maior diante dos demais. Por outro lado, quando o paciente está dormindo sensores que monitoram os movimentos dos membros inferiores podem ter uma prioridade de mensagens baixa.

Vale ressaltar que no funcionamento da rede, dados lidos pelos sensores devem chegar a um sorvedouro e, em algumas situações de sensores mais distantes, diversos saltos de transmissão podem ocorrer para que determinada mensagem atinja seu destino final. Aliado a isso, sensores mais próximos do sorvedouro tendem a receber mais mensagens de diversas fontes distintas. Logo, cabe ao sensor retransmissor decidir qual mensagem ele deve retransmitir primeiro. Neste ponto, a rede se auto-configura, fornecendo a QoS (prioridade) maior às mensagens mais pertinentes ao contexto específico, e assim, favorecendo a entrega momentaneamente destas mensagens. 
Nesse trabalho, tal contexto é ampliado - externando-o a um grau maior de complexidade inserindo outros sensores pertinentes ao objetivo em questão, tais como: sensores de pressão arterial, glicose, oxigênio, inclinação e toxina.

Uma implementação de rede WBAN pode conter diversas características e particularidades devido ao contexto clínico na qual se propõe, conforme é apresentado em [9]. Então, a QoS fornecida deve proporcionar que a tecnologia em questão seja aproveitada ao máximo. Por exemplo, uma entrega eficiente de pacotes reduz possíveis retransmissões, implicando em um menor consumo energético por parte dos nós sensores. Em [10], uma revisão na literatura é realizada, apresentando diversos protocolos para Redes de Sensores Corporais Sem-Fio que levam em conta questões como a conservação de energia, a mobilidade e a QoS na entrega de dados.

Os sensores são alimentados por uma bateria que, respeitando a comodidade do paciente, devem ser pequenas e com uma taxa baixa de substituição ${ }^{1}$. Assim, a avaliação e estudos de métodos que resultam na conservação de energia dos nós sensores são de grande importância [11] [12] [13], pois permitem o aumento da autonomia das RSSFs, e um número menor de intervenções no indivíduo, no caso das WBANs. Essa questão também é considerada no desenvolvimento e análise do algoritmo proposto, denominado nestes trabalho por QoSBody-AODV.

Para fins de avaliação de desempenho do algoritmo proposto neste artigo, foram realizadas simulações utilizando o ambiente Omnet++ [14], onde a rede WBAN transmite dados no padrão de comunicação sem-fio IEEE 802.15.6. ${ }^{2}$ Outrossim, a mobilidade do paciente também foi considerada nos cenários de simulação, o que pode causar desconexões entre sensores vizinhos (e, consequentemente, fim-a-fim). O impacto disso também foi avaliado neste trabalho, onde a movimentação dos sensores, baseou-se em padrões de movimentação humana, implementados pelo MoBAN [15].

O algoritmo proposto (QoSBody-AODV) foi comparado com o algoritmo tradicional AODV que não possui a característica de chaveamento dinâmico de prioridades de mensagens. Através dos resultados obtidos pode-se verificar o quanto o QoSBody-AODV pode auxiliar no número de entrega de pacotes relevantes e na eficiência energética, devido a garantia de QoS provida por ele.

O restante deste artigo está estruturado da seguinte forma: a Seção 2 traz algumas definições de QoS encontradas na literatura; a Seção 3 descreve brevemente o padrão IEEE 802.15.6; a Seção 4 apresenta as principais características do protocolo de roteamento AODV; a proposta deste trabalho é apresentada na Seção 5; a Seção 6 descreve a topologia de rede considerada nos experimentos computacionais; a Seção 7 apresenta o cenário de simulação e discute os resultados; por fim, o trabalho é concluído na Seção 8.

\section{ALGUMAS DEFINIÇÕES DE QOS}

Há na literatura diferentes vertentes para explicitar a QoS nas RSSFs e WBANs. Em [16], por exemplo, considera-se como QoS a procura pelo número ideal de sensores que podem transmitir em um dado momento, buscando a preservação da qualidade do canal de

\footnotetext{
${ }^{1} \mathrm{Em}$ alguns procedimentos de telemedicina necessita-se que o número de trocas de bateria de uma WBAN seja pequeno ou até mesmo inexistente

${ }^{2}$ Em [13], verifica-se que o padrão IEEE 802.15.6 supera o IEEE 802.15.4 na taxa de entrega de dados e na eficiência energética em um cenário WBAN.
}

comunicação $^{3}$. Essa busca pelo número ideal leva em consideração a posição relativa de cada nó na rede, bem como o número de enlaces dos mesmos. Já em [17], os autores consideram as métricas de qualidade de taxa de transmissão, economia de energia, largura de banda e latência como requisitos de QoS.

Sabe-se que, em termos práticos, esses requisitos se diferem, a depender da aplicação WBAN em questão. Porém, os mesmos podem ser especificados em termos de robustez, adaptabilidade, confiabilidade, entre outros [11]. Nesse sentido, os principais desafios da QoS em redes WBAN são: (i) as limitações de recursos, tais como restrição quanto a bateria, largura de banda do canal sem-fio, processamento e memória [18]; (ii) as instabilidades topológicas da rede, como, por exemplo, a necessidade do desligamento de recursos do nó, para fins de economia de energia (modo sleep ${ }^{4}$ ), que o impossibilita de trafegar dados, alterando então o conjunto de sensores ativos em um determinado momento [9]; e, (iii) o tratamento da redundância de dados em um modelo de rede quase sempre dinâmico e heterogêneo (referente ao tipo de informação que nela circula) [20].

\section{O PADRÃO PARA REDE DE SENSORES CORPORAIS SEM-FIO IEEE 802.15.6}

O padrão IEEE 802.15.6 [5] foi criado para atender as particularidades e contextos de uma aplicação WBAN. Sendo assim, o mesmo leva em consideração os efeitos das antenas em diferentes tipos de pessoas [21]. Nessa perspectiva, considera-se que os nós podem estar implantados na pele de um paciente. Por isso, leva-se em consideração as características do tecido humano no processo de transmissão e recepção de dados. Além disso, o padrão provê baixo consumo de energia, uma característica importante para as redes WBANs [22].

O padrão IEEE 802.15.6 considera na camada física, além das ISM usuais, bandas aprovadas por associações médicas [23]. Nesse caminho, há três possibilidades para a implementação da camada física: a NB (Narrow Band), que permite o processo de comunicação em diferentes frequências; a UWB (Ultra Wide Band) que opera na banda centrada em 3993,6 MHz ou 7987,2 MHz; e, por fim, a especificação HBC (Human Body Communications) que utiliza a comunicação de campo elétrico (EFC-Electric Field Communication), suportando transmissão na banda de $21 \mathrm{MHz}$, conforme [24].

Já na subcamada MAC, o padrão também considera, a comunicação com e sem beacon, e o CSMA/CA é adotado como protocolo de acesso ao meio compartilhado. Redes IEEE 802.15.6 possuem um nó, responsável por gerenciar a comunicação em uma topologia em estrela, conforme as redes IEEE 802.15.4. Entretanto, existe a possibilidade de uma topologia em estrela com dois saltos, fornecendo, assim, uma maior escalabilidade para esse tipo de rede; ou seja, um nó pode estar a dois saltos do coordenador da rede.

\footnotetext{
${ }^{3}$ Uma gestão insuficiente implica em perda de energia por excesso de retransmissões dos dados.

${ }^{4}$ Uns dos meios buscados para permitir que esse tipo de rede seja mais eficiente, em relação ao gasto de energia, foi utilizar o Dynamic Power Management (DPM) [19]. O DPM coloca em modo sleep os componentes de hardware que não estão sendo utilizados e usa um escalonador para religa-los
} 


\section{PROTOCOLO AODV}

O AODV é um protocolo de vetor distância, e foi escolhido como base neste trabalho, pois permite que os nós realizem o cálculo de melhor rota sem a necessidade de conhecer toda a topologia da rede, permitindo fácil manutenção e escalabilidade [25]. Além disso, o AODV é reativo; ou seja, suas rotas são criadas sob demanda, preservando os recursos da rede, no que se refere a sua alocação. Dessa forma, consideramos esse protocolo como sendo apropriado para atender WBANs em cenários de alta mobilidade levando em consideração o bom uso de: banda, energia disponível e processamento.

Para se descobrir uma rota, quando um nó sensor de origem precisa encaminhar um pacote para um nó de destino que não consta em sua tabela de roteamento, um processo de descoberta de rotas é realizado. Existe também a manutenção de rotas, na qual pacotes HELLO são direcionados aos enlaces para verificar se os mesmos encontram-se ativos. Se um enlace estiver inativo, uma atualização na tabela de rotas ocorrerá, levando em consideração o atual estado desse nó vizinho.

É válido destacar, ainda, que o AODV possui mecanismos para contornar loops - mesmo desconhecendo a topologia da rede evitando problemas como "contagem até o infinito", contidos em protocolos de vetor distância clássicos. E, além disso, de acordo com [26], o AODV possui resposta rápida às modificações de enlaces dinâmicos, o que tende a permitir uma maior eficiência em cenários de alta mobilidade.

\section{QOSBODY-AODV}

A partir das necessidades e características referentes à QoS em WBANs, propomos o algoritmo QoSBody-AODV, que é uma variação do protocolo de roteamento AODV. O QoSBody-AODV busca garantir a qualidade de serviço da rede atribuindo uma prioridade dinâmica às mensagens, de acordo com o contexto clínico em que o paciente encontra-se inserido. E, para esse processo, não é necessário conhecer toda a topologia da rede, seguindo o mesmo princípio do AODV original.

Além disso, o QoSBody-AODV é responsivo. Isto significa que ele é capaz de mensurar a postura atual do paciente, baseando-se na lista de vizinhos, que é atualizada periodicamente, bem como na variação do sinal medido provocada pelas alterações posturais. Isso é possível e funcional graças a não aleatoriedade dos movimentos dos sensores, conforme visto na Seção 6. A partir disso, o QoSBodyAODV consegue alterar o grau de prioridade das mensagens de acordo com o contexto postural captado pela rede [27].

Essa dinâmica acontece a partir de uma estrutura de dados Heap que gerencia a política de prioridade dos pacotes. Essa estrutura é a responsável por prover a prioridade à entrega de um dado biométrico ao destino, de acordo com um contexto clínico previamente definido. Exemplos de ordem de prioridade para as atividades do cotidiano e tratamento clínico são apresentados nas Tabelas 1 e 2, respectivamente.

Nas tabelas temos as colunas "Atividade" e "Tratamento", que indicam a ordem de prioridades que será atribuída às mensagens dos sensores aderidos ao corpo do paciente/usuário. Por exemplo, digamos que a avaliação da postura, realizada pelo QoSBody-AODV, estime que o paciente esteja "dormindo"; neste caso as mensagens dos sensores ECG terão prioridade em relação as mensagens geradas pelo sensores de Glicose, conforme a tabela.

Esta ordem de prioridades também pode ser definida de forma fixa, ou seja, caso seja interessante realizarmos um levantamento ou avaliação cardiovascular no paciente/usuário, durante todo o funcionamento do protocolo proposto a ordem de prioridades das mensagens dos sensores (ECG, Pressão, Glicose e Movimento) não será alterada, independente da mudança de postura praticada pelo paciente/usuário.

Estes modos de operação são relevantes quanto a necessidade de avaliação realizada ao usuário/paciente.

Tabela 1: Ordem de Prioridade dos Sensores para Atividade do Cotidiano.

\begin{tabular}{|l|c|}
\hline Atividade & \multicolumn{1}{|c|}{ Ordem de Prioridade } \\
\hline Dormir & ECG, Pressão, Oxigênio e Glicose \\
\hline Cotidiano & ECG, Pressão, Oxigênio e Inclinação \\
\hline Andar & ECG, Pressão, EMG e Movimento \\
\hline Correr & ECG, Pressão, Movimento e Lático \\
\hline
\end{tabular}

Tabela 2: Ordem de Prioridade dos Sensores para Tratamento Clínico.

\begin{tabular}{|l|c|}
\hline \multicolumn{1}{|c|}{ Tratamento } & Ordem de Prioridade \\
\hline Cardiovascular & ECG, Pressão, Glicose e Movimento \\
\hline Oncológico & Pressão, ECG, Glicose e Oxigênio \\
\hline Diabetes & Glicose, ECG, Pressão e Oxigênio \\
\hline
\end{tabular}

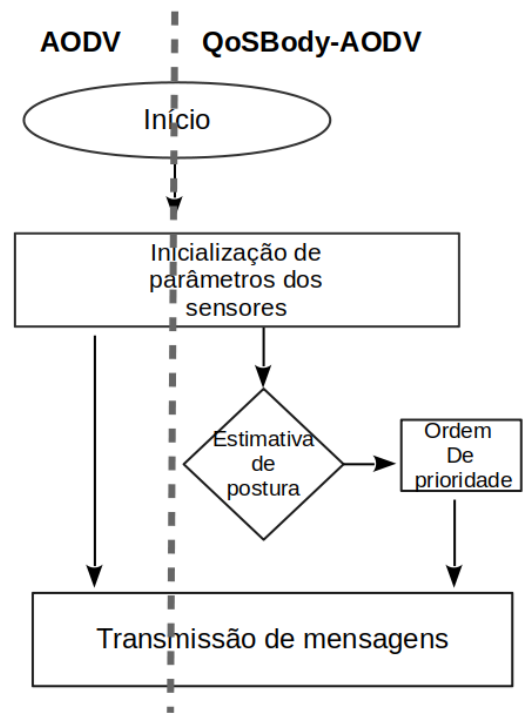

Figura 1: Funcionamento: AODV x QoSBody-AODV. 
Conforme apresentado na Figura 1, há exemplificado, de forma simplificada, o funcionamento do protocolo proposto (QosBodyAODV) comparado ao AODV original. O QosBody-AODV, através do sua estimativa de postura, ou mesmo do modo de operação clínico apresentado na Tabela 2, verifica qual a postura atual do usuário/paciente e, a partir disso, atribui prioridades diferentes às mensagens geradas por cada sensor considerado.

\section{TOPOLOGIA DE REDE WBAN}

A distribuição dos sensores no cenário estabelecido foi baseada nas propostas apresentadas em [28] e [29], em que leva-se em consideração os pontos preferíveis para a coleta dos dados biométricos. Além disso, a topologia de rede WBAN proposta nesse trabalho vista na Figura 2 - possui um padrão de movimento baseado em posturas típicas do ser humano [15]. Esta adoção visa obtermos resultados mais próximos da realidade.

Um modelo de locomobilidade adequado para redes WBANs deve ser capaz de modelar estatisticamente os padrões de movimentos corretos dos nós individuais instalados em um corpo, bem como o movimento do próprio corpo humano. Ao mesmo tempo, tal modelo deve ser adaptável a vários cenários de aplicação; ou seja, para as mais diversas atividades humanas.

Nesse sentido, o padrão utilizado foi implementado a partir da inserção de arquivos contendo amostras de movimentação típica do ser humano - ditando como cada sensor deve se comportar segundo o projeto MoBAN [15]. Estes padrões de mobilidade foram lidos e executados no ambiente chamado Omnet++ [14].

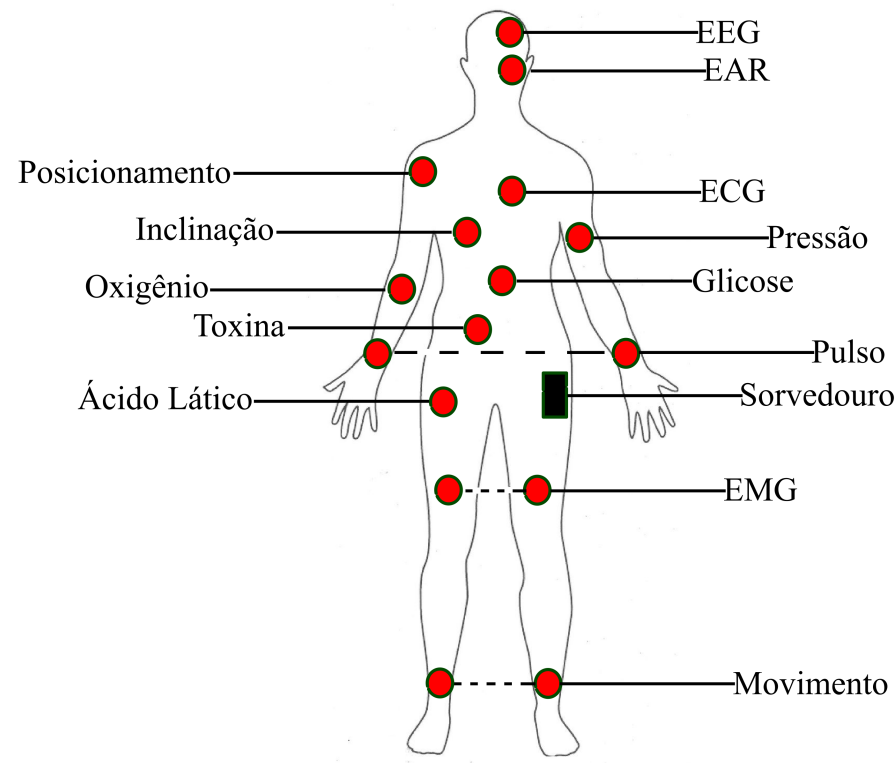

Figura 2: Topologia de rede considerada nas simulações.

\section{SIMULAÇÕES E RESULTADOS}

Considerou-se o ambiente de simulação Castalia/MoBAN baseado no simulador a eventos discretos Omnet++. O desempenho do algoritmo proposto foi avaliado sobre o padrão de rede sem-fio IEEE
802.15.6. O esquema de rede foi submetido ao padrão de movimentação baseado em posturas típicas do ser humano, a fim de obter resultados mais próximos da realidade.

Os gráficos apresentam os resultados médios de 30 rodadas de simulação, com o intervalo de confiança de 95\%, A Figura 2 - seção 6 - apresenta a topologia de rede utilizada nas simulações. Considerase como sorvedouro, um aparelho robusto externo ao corpo, um smartphone, a título de exemplo, que possui energia residual suficiente para funcionar ao longo de todo tempo de vida da rede. A energia inicial dos nós sensores é de 18.720 Joules, quantidade de energia típica de duas baterias AA [30], a taxa de transmissão padrão é de 5 pacotes/segundo, onde cada pacote possui 100 Bytes de tamanho, com um buffer de 32 frames, a potência de transmissão é de $-10 \mathrm{dBm}$ e a sensibilidade do receptor é de $-87 \mathrm{dBm}$. Esses parâmetros utilizados foram baseados nos parâmetros apresentados em [31].

As simulações foram realizadas tomando como base as prioridades definidas nas Tabelas ${ }^{5} 1$ e 2 . Por comodidade e tamanho das tabelas apenas os quatro primeiros sensores da fila são apresentados. Considerou-se na avaliação, o contexto de um tratamento cardiovascular (Tabela 2), levando-se em consideração que o paciente poderá realizar todas as atividades apresentadas na Tabela 1.

De modo a avaliar o impacto do algoritmo proposto sua eficiência em relação à entrega de pacotes, dois cenários foram estudados:

(1) AODV e QoSBody-AODV: neste primeiro cenário, pode ocorrer a morte de alguns dos nós presentes na rede, ou seja instante em que a energia de suas baterias acaba.

(2) AODV $^{*}$ e QoSBody-AODV*: neste segundo cenário, considera-se que a energia inicial dos nós presentes na rede foi configurada com um valor suficientemente alto, de modo que todos os nós permaneçam ativos durante todo o tempo de simulação.

Podemos verificar a comparação entre esses dois cenários nos resultados apresentados nas Figuras 4, 5 e 6.

\subsection{Avaliação do Consumo de Energia}

A Figura 3 apresenta o consumo de energia total por nó sensor para as primeiras 24 horas de funcionamento da rede. Esse período é suficiente para que a rede (neste caso o usuário/paciente) experimente todos os possíveis estados posturais considerados nas Tabelas 1 e 2, e desta forma a avaliação do algoritmo proposto seja ampla.

Nesse experimento, comparamos o protocolo AODV original com o QoSBody-AODV e, desta maneira, podemos avaliar quanto de redução no consumo de energia o algoritmo proposto obteve em relação ao original. Verifica-se um melhor gerenciamento de energia pelo algoritmo proposto, pois o mesmo fornece condições favoráveis para a transmissão eficiente dos dados de um determinado sensor, através de uma melhor gestão do canal, mitigando retransmissões, de acordo com seu grau de prioridade.

\footnotetext{
${ }^{5}$ Nota-se, a priori, grande prioridade do sensor ECG em todas as atividades relatadas, seguido pelo sensor de pressão. Os sensores EMG (tração muscular) e Movimento são
} levados em consideração nas atividades de andar e correr. 


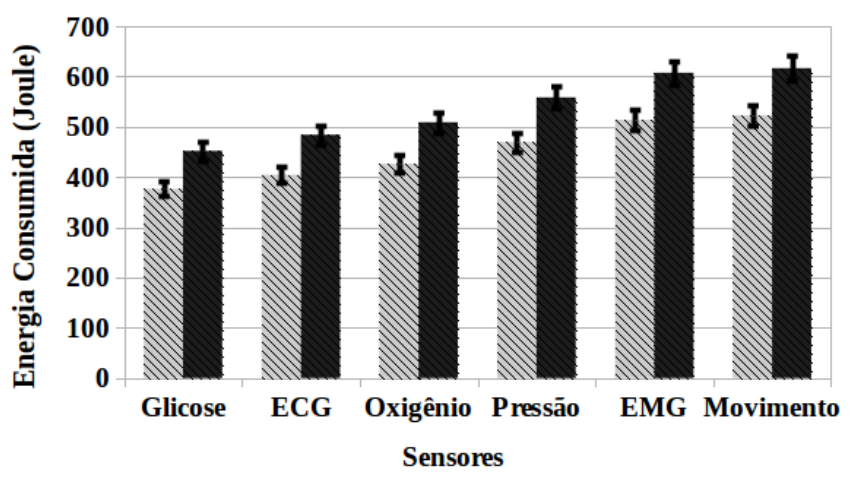

W QoSBody-AODV AODV

Figura 3: Consumo de energia total por nó em um período de 24 horas.

\subsection{Avaliação da Eficiência na Entrega de Pacotes}

A Figura 4 apresenta a taxa de entrega de pacotes nas últimas 24 horas de funcionamento da rede. Associou-se, ainda, os estados posturais ocorridos nesse período (descritos na Tabela 1). Vale ressaltar que, normalmente, esse é o período mais crítico no funcionamento de uma rede WBAN. É nesse período que ocorrem as primeiras mortes ${ }^{6}$ dos sensores intermediários, tornando mais crítica a entrega de pacotes. É nele que ocorre a menor taxa de entrega de pacotes, além de um maior consumo de energia devido às retransmissões, causadas pela redução significativa da energia residual de alguns sensores, bem como sua morte. De modo a avaliar essa questão, comparamos o cenário em que os nós sensores podem exaurir sua bateria (AODV e QoSBody-AODV) com o cenário com energia inicial suficiente para que não haja morte de nenhum sensor (AODV* e QoSBody-AODV*).

Conforme esperado, há uma menor taxa de entrega no cenário em que há morte de nós sensores, e isso se evidencia mais acentuadamente nas últimas horas de simulação. Além disso, há um aumento na taxa de entrega para o cenário em que não há mortes de sensores. Isto ocorre porque neste período final, o paciente está dormindo, reduzindo assim a demanda de alguns sensores e, consequentemente, a competição pelo meio. Por fim, pode-se verificar que o algoritmo proposto supera o AODV original em ambos os cenários.

Em geral, WBANs atuam com pequenas taxas de transmissão de pacotes, conforme definido na Seção 7. Nesse contexto, a Figura 5 apresenta a perda média de pacotes ao longo do tempo de funcionamento da rede, medida após $24 h$ de simulação, para diferentes taxas de transmissão (em pacotes por segundo), além da taxa padrão de 5 pacotes por segundo. O objetivo é avaliar o comportamento do algoritmo proposto em cenários onde existe a necessidade de uma maior taxa de transmissão. Pode-se verificar que há um menor percentual de perdas quando o esquema de prioridade de mensagens é utilizado, mesmo em situações de mais alta taxa de transmissão.

${ }^{6}$ Morte do sensor é um termo utilizado para descrever que o mesmo esgotou a energia residual de sua bateria, conforme [32].

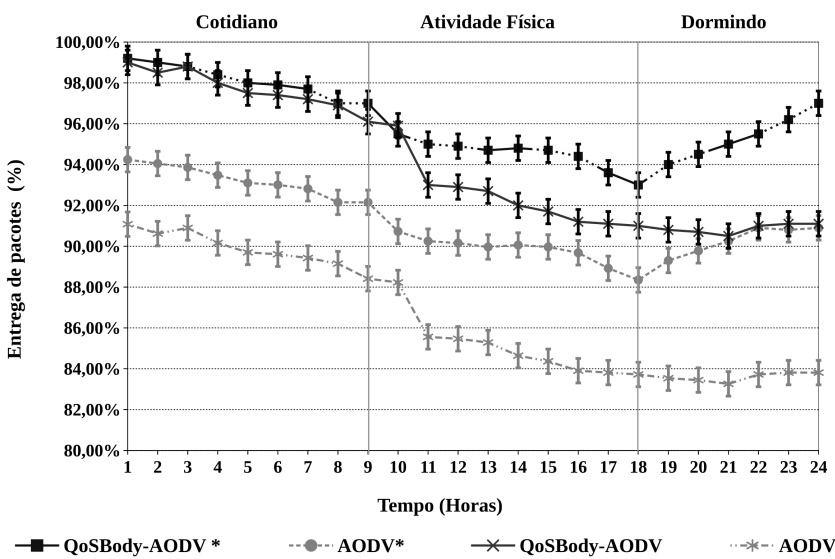

Figura 4: Taxa de entrega de pacotes nas últimas 24 horas de funcionamento da rede. Comparou-se o cenário em que pode haver morte de sensores (denotado por AODV e QoSBody-AODV) com o cenário em que a energia inicial dos nós é suficiente para que não haja mortes de nós sensores durante a simulação (denotado por $\mathrm{AODV}^{*}$ e QoSBody-AODV).

Outrossim, verifica-se uma menor taxa de perdas no cenário em que não há morte de sensores.

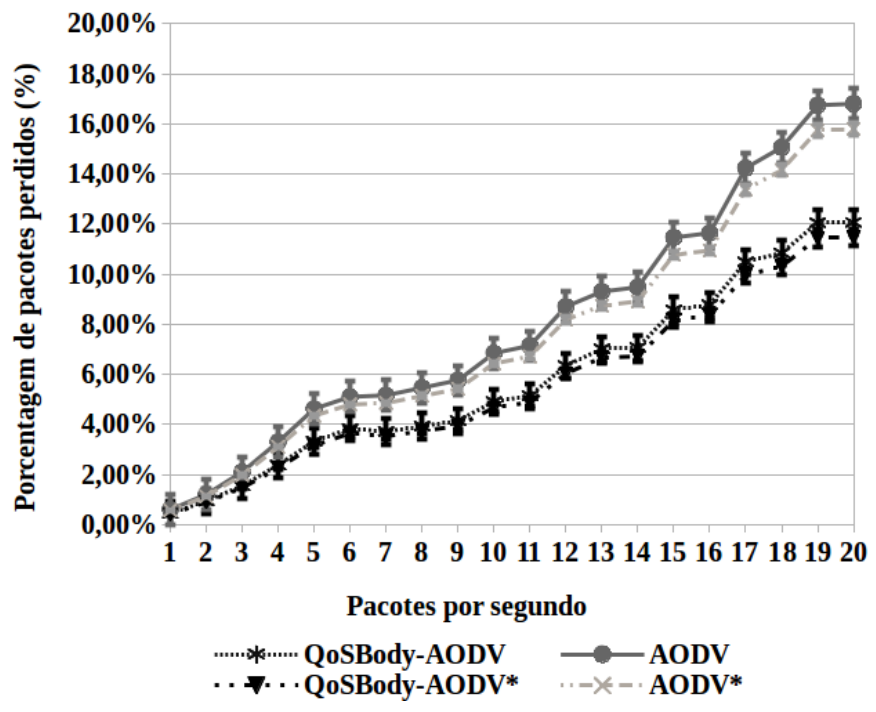

Figura 5: Perda de pacotes após $24 \mathrm{~h}$ de simulação, para diferentes taxas de transmissão (em pacotes por segundo). Comparou-se o cenário em que pode ocorrer morte de sensores (AODV e QoSBody-AODV) com o cenário em que a energia inicial dos nós é suficiente para que não haja mortes de nós sensores durante a simulação (AODV* e QoSBodyAODV $^{*}$ ).

Verificou-se ainda, na Figura 6, o atraso médio para a entrega dos pacotes, para os sensores de ECG, Pressão, Glicose e Movimento (tratamento cardiovascular), após 24 horas de simulação. Pôde-se 
observar que o algoritmo proposto apresenta um menor atraso médio na entrega de pacotes, para todos os sensores apresentados ${ }^{7}$, quando comparado com o algoritmo original.

É válido enfatizar que o ocorrido deve-se ao fato de o QoSBodyAODV apresentar uma melhor gestão do canal de comunicação, implicando em uma maior disponibilidade do meio, para o envio de pacotes até o referido destino. Além disso, verifica-se um atraso menor quando não há morte de nenhum nó da rede, corroborando aos resultados anteriormente apresentados.

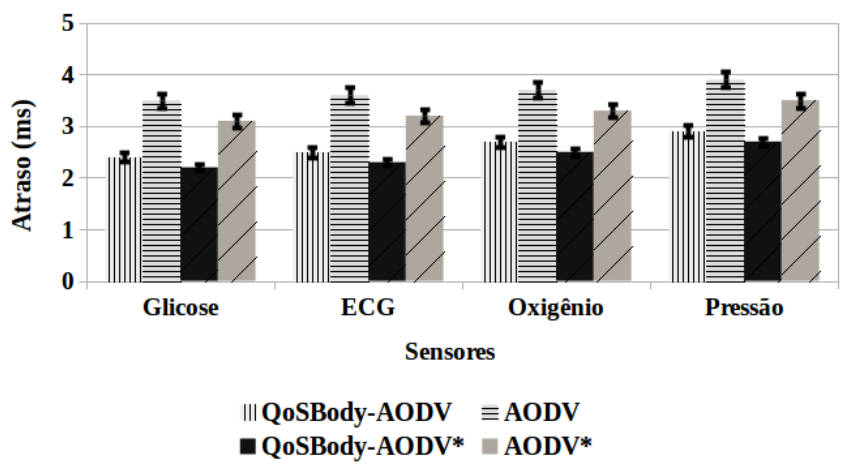

Figura 6: Atraso médio na entrega de pacotes após $24 \mathrm{~h}$ de simulação. Comparou-se o cenário em que pode haver morte de sensores (AODV e QoSBody-AODV) com o cenário em que a energia inicial dos nós é suficiente para que não haja mortes de nós sensores durante a simulação $\left(\mathrm{AODV}^{*}\right.$ e QoSBodyAODV $^{*}$ ).

Por fim, a Figura 7 apresenta a porcentagem de perda de pacotes por transbordamento de dados - buffer overflow - que ocorre pela incapacidade dos nós sensores em processar e rotear um dado número de pacotes em um curto período de tempo. Nota-se, que o algoritmo proposto permite uma melhor gestão da unidade de processamento dos sensores. Novamente, isso se dá pela melhor gestão do canal de comunicação.

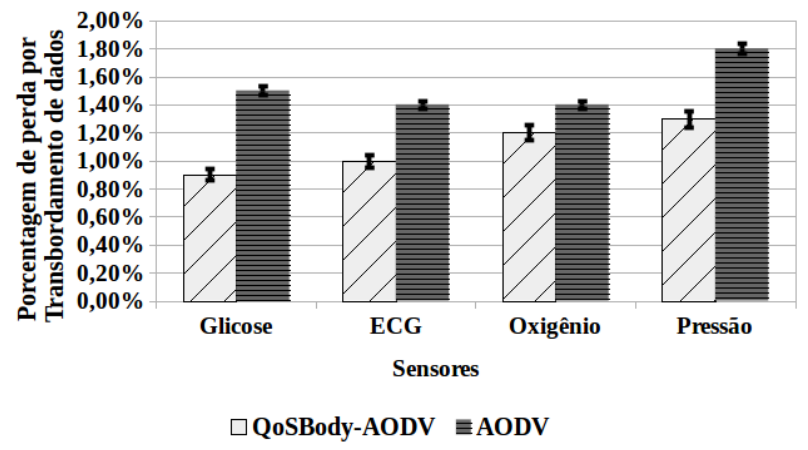

Figura 7: Porcentagem de perda de pacotes por transbordamento de dados - buffer overflow - nas últimas 24 horas de funcionamento da rede. Levou-se em consideração o cenário em que pode ocorrer morte de sensores.

\footnotetext{
${ }^{7} \mathrm{O}$ comportamento se manteve para os demais sensores da rede.
}

\section{CONCLUSÕES}

Neste trabalho, propomos o algoritmo QoSBody-AODV, que fornece QoS (Qualidade de Serviço) para Redes de Sensores Corporais SemFio (WBANs), de modo a garantir a entrega de pacotes baseada no grau de prioridade atribuído ao sensor. Essa atribuição permite uma melhor gestão do meio de comunicação, reduzindo o consumo de recursos da rede como, por exemplo, o gasto de energia nas retransmissões.

O algoritmo proposto se diferencia do AODV (original), pois inclui funções que levam em consideração o contexto da rede e a prioridade de cada nó para traçar rotas da origem até o destino. $\mathrm{O}$ algoritmo é executado sobre o padrão IEEE 802.15.6 e foi comparado com o protocolo de roteamento AODV. Estudos de caso comparando um cenário em que pode ocorrer morte de sensores com um cenário em que a energia inicial dos nós sensores é suficiente para todo período de simulação $(24 \mathrm{~h})$ foram realizados. Os resultados obtidos mostram que o QoSBody-AODV consegue uma economia de energia em relação ao AODV original, além de ter um menor atraso e menor taxa de perda de pacotes, mesmo quando um cenário com mobilidade é considerado.

\section{AGRADECIMENTOS}

Os autores agradecem o apoio da DIPPG-CEFET/RJ.

\section{REFERÊNCIAS}

[1] A. F. L. Loureiro, J. M. S. Nogueira, R. B. Ruiz, R. A. F. Mini, E. F. Nakamura, and M. S. Figueiredo. Redes de Sensores Sem Fio. In Minicurso do Simpósio Brasileiro de Redes de Computadores, pages 179-226, 2003.

[2] I. F. Akyildiz, W. Su, Y. Sankarasubramaniam, and E. Cayirci. Wireless Sensor Networks: A Survey. Computer Networks, 38(4):393-422, March 2002.

[3] F. R. Henriques, L. C. S. C. Retondaro, E. F. Carneiro, and D. K. T. de Moraes. Remote Monitoring of Electrocardiogram Signals Transmitted in a ZigBee Network. In Proceedings of the XXXI Simpósio Brasileiro de Telecomunicações-SBrT 2013, pages 1-5, Fortaleza-CE, 2013.

[4] P. Baronti, P. Pillai, V. Chook, S. Chessa, A. Gotta, and Y. F. Hu. Wireless Sensor Networks: a Survey on the State of Art and the 802.15.4 and ZigBee Standards. Computer Communications, 30(7):1655-1695, May 2007.

[5] IEEE Standard for Local and metropolitan area networks - Part 15.6: Wireless Body Area Networks. In Institute of Electrical and Electronic Engineers, New York, USA, Standard, 2012.

[6] Vinicius C Ferreira, Débora C Muchaluat-Saade, and Célio VN de Albuquerque. Estudo sobre estabilidade de rotas em redes corporais sem fio. In Escola Regional de Computação Aplicada à Saúde (ERCAS-R7 2018), volume 6. SBC, 2018.

[7] Shubhangi Sonone and Vivek KR. Sharma. Improved new aodv (inaodv) routing protocol for collision free wireless sensor body area network for health monitoring. International fournal of Computer Science and Information Technologies, 5 (1), 2014.

[8] M. Ambigavathi and D. Sridharan. Priority based aodv routing protocol for critical data in wireless body area network. In 2015 3rd International Conference on Signal Processing, Communication and Networking (ICSCN), pages 1-5, 2015.

[9] C. B. M. Ferreira, R. M. Guedes, and F. R. Henriques. Cluster-Head Switching Algorithm Based on Node Temperature in Wireless Body Sensors Networks. In Anais do Simpósio Brasileiro de Sistemas Multimídia e Web (WEBMEDIA), Rio de Janeiro-RJ, 2019.

[10] Amira Meharouech, Jocelyne Elias, and Ahmed Mehaoua. Moving towards bodyto-body sensor networks for ubiquitous applications: A survey. F. Sens. Actuator Netw., 8(2), 2019.

[11] F. R. Henriques, L. Lovisolo, and M. G. Rubinstein. DECA: distributed energy conservation algorithm for process reconstruction with bounded relative error in wireless sensor networks. EURASIP fournal on Wireless Communications and Networking, 2016(163):1-18, July 2016.

[12] Felipe Da Rocha Henriques, Lisandro Lovisolo, and Eduardo Antônio Barros da Silva. Rate-distortion performance and incremental transmission scheme of compressive sensed measurements in wireless sensor networks. Sensors, 19(2), 2019. ISSN 1424-8220. doi: 10.3390/s19020266. URL http://www.mdpi.com/1424$8220 / 19 / 2 / 266$.

[13] C. B. M. Ferreira and F. R. Henriques. Chaveamento de Transmissão Baseado na Temperatura do Nó em Redes de Sensores Corporais Sem Fio. In Anais do 
XXXVI Simpósio Brasileiro de Telecomunicações e Processamento de Sinais-SBrT 2018, pages 1-5, Campina Grande-PB, 2018.

[14] Andras Varga. Omnet++. In Modeling and tools for network simulation, pages 35-59. Springer, 2010.

[15] Majid Nabi, Marc Geilen, and Twan Basten. Moban: A configurable mobility model for wireless body area networks. In Proceedings of the 4th International ICST Conference on Simulation Tools and Techniques, pages 168-177. ICST (Institute for Computer Sciences, Social-Informatics and ..., 2011.

[16] Aura Ganz, Zvi Ganz, and Kitti Wongthavarawat. Multimedia Wireless Networks: Technologies, Standards and QoS. Pearson Education, 2003.

[17] MA Ameen, Ahsanun Nessa, and Kyung Sup Kwak. Qos issues with focus on wireless body area networks. In 2008 Third International Conference on Convergence and Hybrid Information Technology, volume 1, pages 801-807. IEEE, 2008.

[18] Yuanli Wang, Xianghui Liu, and Jianping Yin. Requirements of quality of service in wireless sensor network. In International Conference on Networking, International Conference on Systems and International Conference on Mobile Communications and Learning Technologies (ICNICONSMCL'06), pages 116-116. IEEE, 2006.

[19] Amit Sinha and Anantha Chandrakasan. Dynamic power management in wireless sensor networks. IEEE Design \& Test of Computers, 18(2):62-74, 2001.

[20] Lars Wolf and Sana Saadaoui. Architecture concept of a wireless body area sensor network for health monitoring of elderly people. In 2007 4th IEEE Consumer Communications and Networking Conference, pages 722-726. IEEE, 2007.

[21] Husnu Yalduz, Burak Koç, Lokman Kuzu, and Mustafa Turkmen. An ultra-wide band low-sar flexible metasurface-enabled antenna for wban applications. Applied Physics A, 125(9):609, 2019.

[22] Chenfu Yi, Lili Wang, and Ye Li. Energy efficient transmission approach for wban based on threshold distance. IEEE sensors journal, 15(9):5133-5141, 2015.

[23] Kyung Sup Kwak, Sana Ullah, and Niamat Ullah. An overview of ieee 802.15. 6 standard. In 2010 3rd International Symposium on Applied Sciences in Biomedical and Communication Technologies (ISABEL 2010), pages 1-6. IEEE, 2010.
[24] V. C. Ferreira, H. Balbi, F. L. Seixas, C. Albuquerque, and D. C. Muchaluat-Saade. Wireless Body Area Networks: An Overview. In Minicurso do XXXV Simpósio Brasileiro de Telecomunicações e Processamento de Sinais-SBrT 2017, pages 1-14, São Pedro-SP, 2017.

[25] Clement Ogugua Asogwa, Xiaoming Zhang, Degui Xiao, and Ahmed Hamed. Experimental analysis of aodv, dsr and dsdv protocols based on wireless body area network. In Internet of Things, pages 183-191. Springer, 2012.

[26] Charles E Perkins and Elizabeth M Royer. Ad-hoc on-demand distance vector routing. In Proceedings WMCSA'99. Second IEEE Workshop on Mobile Computing Systems and Applications, pages 90-100. IEEE, 1999.

[27] Syed Muhammad Khaliq-ur-Rahman Raazi, Heejo Lee, Sungyoung Lee, and Young-Koo Lee. Bari+: a biometric based distributed key management approach for wireless body area networks. Sensors, 10(4):3911-3933, 2010.

[28] Li Huang, Maryam Ashouei, Firat Yazicioglu, Julien Penders, Ruud Vullers, Guido Dolmans, Patrick Merken, Jos Huisken, Harmke de Groot, Chris Van Hoof, et al. Ultra-low power sensor design for wireless body area networks: challenges, potential solutions, and applications. International Journal of Digital Content Technology and its Applications, 3(3):136-148, 2009.

[29] Monika Verma and Rajesh Rai. Energy-efficient cluster-based mechanism for wban communications for healthcare applications. International fournal of Computer Applications, 120(19), 2015.

[30] Athanassios Boulis. Castalia user's manual. NICTA. Marzo del, 2011

[31] A. Bouayad, E. H. Chaoui, M. E. Ghazi, and M. E. Bekkali. Energy Efficiency of IEEE 802.15.6 MAC Access Modes for Remote Patient Monitoring Applications. International fournal of Computer Science and Information Security, 13(4):68-77, Apr 2015.

[32] Haroon Rashid and Ashok Kumar Turuk. Dead reckoning localisation technique for mobile wireless sensor networks. IET Wireless Sensor Systems, 5(2):87-96, 2015. 